\title{
In Vivo Light-Driven DNA Binding and Cellular Uptake of Nucleic Acid Stains
}

Mateo I. Sánchez, José Martínez-Costas, Francisco Gonzalez, María A. Bermudez, M. Eugenio Vázquez, and José L. Mascareñas

\section{Accepted Manuscript}

This document is the Accepted Manuscript version of a Published Work that appeared in final form in ACS Chemical Biology, copyright (C) American Chemical Society after peer review and technical editing by the publisher. To access the final edited and published work see: https://doi.org/10.1021/cb300100r

\section{How to cite:}

Sánchez, M., Martínez-Costas, J., Gonzalez, F., Bermudez, M., Vázquez, M., Mascareñas, J. (2012). In Vivo Light-Driven DNA Binding and Cellular Uptake of Nucleic Acid Stains. ACS Chem. Biol., 7 (7), 1276-1280. DOI: 10.1021/cb300100r

\section{Copyright information:}

(C) 2012 American Chemical Society 


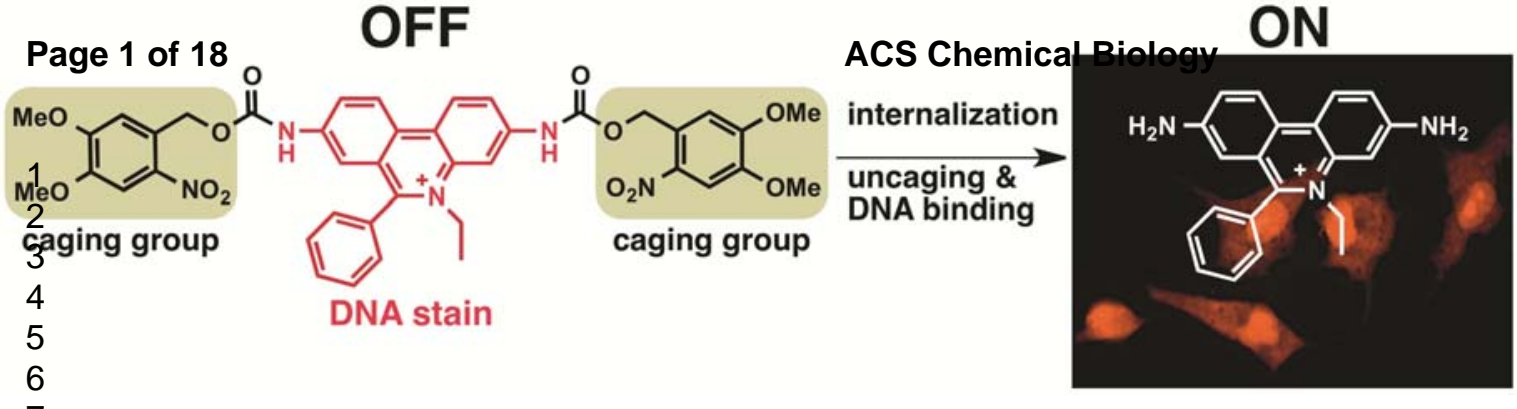

7

9

10

11

12

13

14

15

16

17

18

19

20

21 


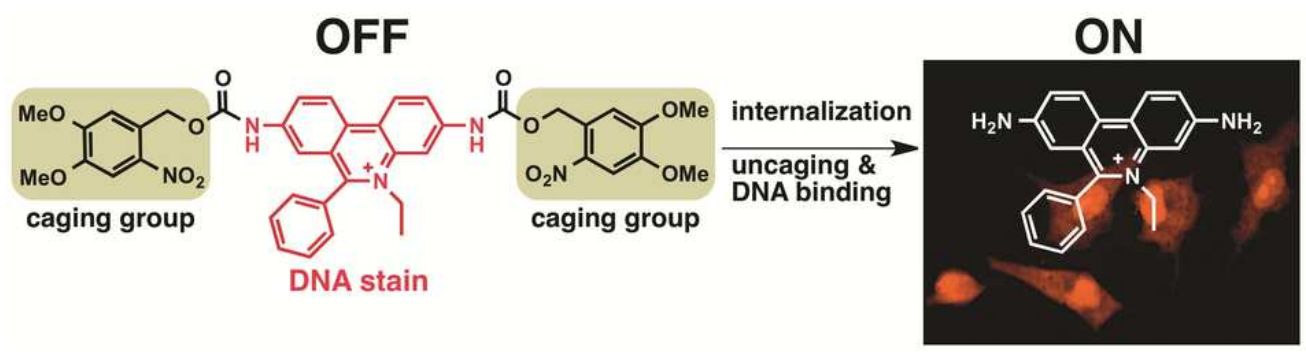

$40 \times 10 \mathrm{~mm}(600 \times 600$ DPI $)$ 


\title{
In vivo light-driven DNA binding and cellular uptake of DNA stains
}

Mateo I. Sánchez, ${ }^{\S}$ José Martínez-Costas, ${ }^{\top} F r a n c i s c o$ Gonzalez, ${ }^{\dagger, \Delta}$ Maria A. Bermudez, ${ }^{\dagger} M$. Eugenio Vázquez, ${ }^{\S, *}$ and José L. Mascareñas ${ }^{\S, *}$

$\S$ Departamento de Química Orgánica and ๆ Departamento de Bioquímica y Biología Molecular, Centro Singular de Investigación en Química Biológica y Materiales Moleculares y Unidad Asociada al CSIC.

† Departamento de Fisiología. Universidad de Santiago de Compostela, 15782 Santiago de Compostela, Spain.

${ }^{\Delta}$ Servicio de Oftalmologia, Complejo Hospitalario Universitario de Santiago de Compostela, 15705 Santiago de Compostela, Spain.

joseluis.mascarenas@usc.es, eugenio.vazquez@usc.es

\begin{abstract}
Chemical derivatization of DNA stains such as ethidium bromide or DAPIwith tailored, photoresponsive caging groups, allows for "on demand" spatiotemporal control of their "in vivo" DNA binding, as well as for improving their cellular uptake. This effect was particularly noteworthy for a Nvoc-caged derivative of ethidium bromide that, in contrast with the parent stain, is effectively internalized into living cells. The activation strategy works in light-accessible, therapeutically relevant settings, such as human retinas, and can be even applied for the release of active compounds in the eyes of living mice.
\end{abstract}


The development of nucleic acid-targeted drugs continues to be a major research endeavor at the interface between chemistry and biomedicine. A large variety of DNA-binding agents, have been developed, and many of them have found application as drugs or as DNA stains. ${ }^{1,2,3,4,5}$ Unfortunately, many of these molecules present selectivity and toxicity problems that seriously restrict their therapeutic potential. In this context, derivatizing these molecules with photolabile appendages that confer advantageous physicochemical features while providing for external control of their activity might open important therapeutic avenues. ${ }^{6,7,8}$ Needless to say that the use of light-activated compounds in photodynamic therapy might greatly benefit from current technical advances that make possible to irradiate almost any tissue in the human body. ${ }^{9}$

DAPI (4',6-diamidino-2-phenylindole, 1) and ethidium bromide $(\mathrm{EtBr}, 3,8-d i a m i n o-5-e t h y l-6-$ phenyl-phenanthridinium bromide, 2) are among the best known DNA stains,,$^{10,11,12,13,14}$ and have found wide and important applications in molecular and cell biology. Ethidium bromide binds DNA by intercalation, and has been extensively used for detecting double stranded DNA, mainly in gel electrophoresis assays. However, it cannot be used for direct staining of cellular nucleic acids, as it is poorly internalized. In contrast, the blue-fluorescent DAPI can be used to stain cell nuclei and, unlike the non-specific $\mathrm{EtBr}$, it prefers to bind $\mathrm{A} / \mathrm{T}$-rich $\mathrm{DNA}$ sequences, inserting into their narrow minor groove.

We have recently demonstrated that caging of the amidinium moieties of DAPI and other bisbenzamidinium DNA binders with suitable photoresponsive protecting groups suppresses their DNA binding..$^{15}$ Importantly, irradiation with UV light removes the caging groups and regenerates the parent, active compounds, thus allowing the control of the in vitro DNA binding activity of these molecules by external stimuli (UV light). We were then challenged to demonstrate that such conditional activation strategy could also be used in living cells and tissues, a process that should be easily monitored by the increased fluorescence of these 
molecules upon staining of the cell nuclei. In addition, we were also intrigued by the possibility of extending the caging strategy to other DNA binders lacking amidinium groups, such as ethidium bromide $(\mathrm{EtBr})$.

Herein we demonstrate that both, DAPI (1) and $\mathrm{EtBr}$ (2), can be efficiently caged using photolabile groups, and that their DNA binding can be restored in living cells and in therapeutically-relevant tissues such as retinas, in an spatially and temporarily-controlled manner. We also show that it is even possible to release the DNA stains in the eyes of living mice by using an external irradiation source.

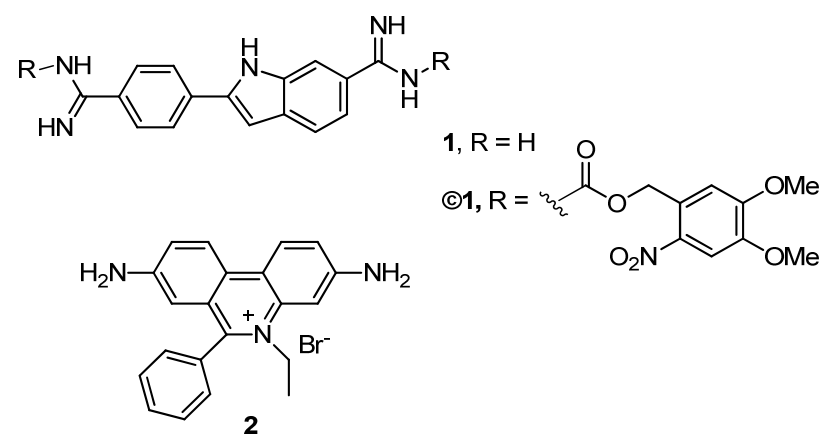

Figure 1.Structures of DAPI (1), its caged derivative (@1) and ethidium bromide (2).

As expected from the relatively low pKa of the amidines and their propensity to act as leaving groups, the caging groups of DAPI derivative $\odot 1^{15}$ can be readily removed in presence of dsDNAs, in just 1.5-2 min, using a standard gel transilluminator lamp as irradiation source $(\lambda=300$ to $375 \mathrm{~nm}$, see the supporting information, Figure S1). For the in vivo assays, we incubated independent samples of Vero cells, ${ }^{16}$ with $5 \mu \mathrm{M} \odot 1$ for 30 minutes, and monitored their evolution by fluorescence microscopy. Initial irradiation experiments were carried out using the transilluminator, but the UV light source from the microscope could also effectively photolyze $@ 1$. This allowed an easy monitoring of the changes in fluorescence emission of the dye inside the cells in real time. Remarkably, the fluorescence emission is initially relatively weak and diffuse, 
but apparently more intense on the perimeter (membrane) of the cells. Over time it becomes progressively concentrated in the cell nuclei, while growing fainter at the cell edges. Indeed, after 20 minutes under the microscope, the edges become barely distinguishable and only the nuclei are observed (see time-lapse video S1 in the supporting information). Control experiments with liposomes confirm that, as expected, @1 1 is basically non-emissive, however addition of DAPI to a liposome solution results in a marked increase in its emission intensity (see Supporting Information, page S11). Therefore it can be safely stated that the fluorescence observed in the membrane is due to uncaged DAPI.

In order to separate the effects of the uncaging and cellular redistribution, Vero cells were incubated with $\odot 1$ in PBS for $30 \mathrm{~min}$, and irradiated for $2 \mathrm{~min}$ in the transilluminator (enough to uncage most of the stain). Comparison of photographs obtained immediately after the irradiation, and after five minutes, confirms that the uncaged dye requires some time to accumulate and stain the cell nuclei (see supporting information, page S16). All these results are consistent with the presence of the hydrophobic caged DAPI (৫1) in the cytoplasmic membrane and probably also in other cell locations. Upon irradiation, photolysis of $\odot 1$ releases the fluorogenic DAPI, which is highly emissive in the hydrophobic phospholipid matrix. However, the uncaged DAPI is very hydrophilic (ACD/Labs LogD $(\mathrm{pH} 7.4)=-1.03),{ }^{17,18}$ and is therefore driven out of the membrane and freely diffuse towards the cell nuclei, finally forming a stable, fluorescent DNA complex. 

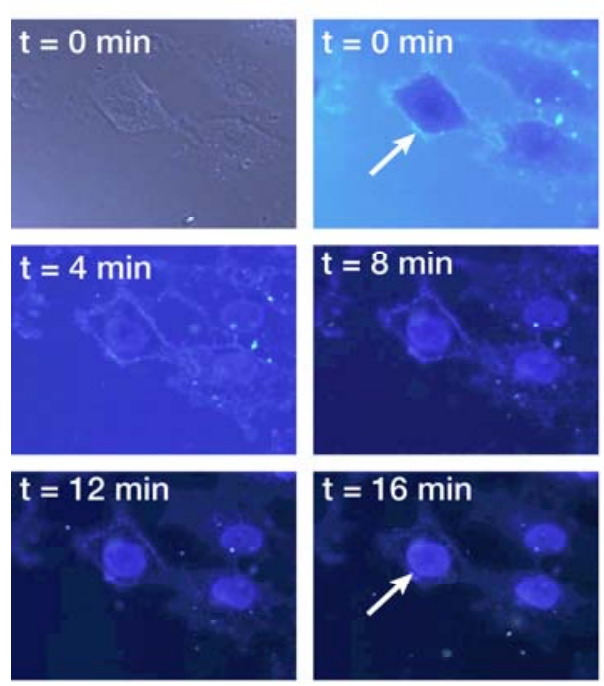

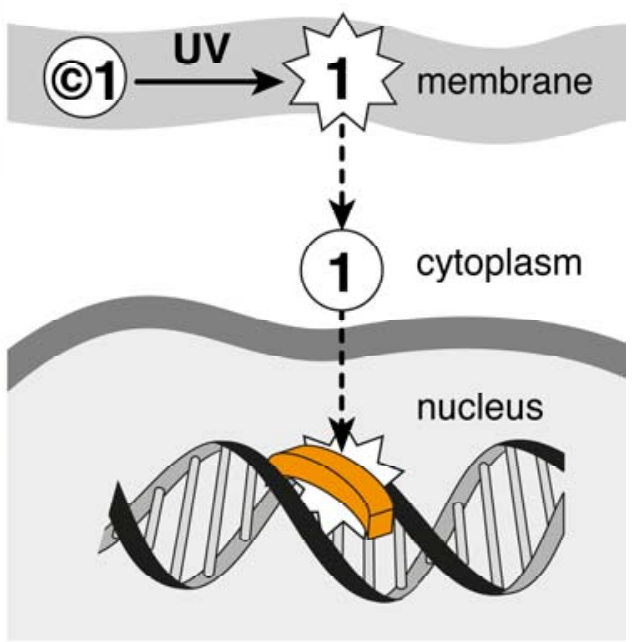

Figure 2. Left: Fluorescence microscopy of Vero cells incubated with $5.0 \mu \mathrm{M} @ 1$ showing the fluorescence changes inside the cells ( $t=0$ : brightfield and fluorescence; $t=4 \mathrm{~min}$ to $t=16 \mathrm{~min}$ : fluorescence); arrows highlight the brighter areas. Right: Schematic representation of the proposed activation dynamics of caged DAPI, which is, at least in part, stored in the cell membrane. Initial background fluorescence at $t=0$, most probably results from non-specific interaction between uncaged DAPI and the hydrophobic polymeric Mowio/ $₫$ matrix used as coverslip mounting solution.

Once demonstrated the possibility of in vivo UV-controlled DNA interaction with a minor groove binder, we sought to extend this strategy to $\operatorname{EtBr}(2)$, which binds DNA by intercalation and lacks the amidinium groups of DAPI. Despite its wide use as an in vitro DNA stain, EtBr has very limited value for in vivo experiments due to its poor cell membrane permeability. We envisioned that masking the amino groups of EtBr with the non-polar Nvoc caging group might not only interfere with its DNA binding, but also improve the internalization properties of the stain by increasing its hydrophobic character. Therefore the bis-Nvocphenantridyl derivative $\subseteq 2$ was prepared by treatment of the commercial EtBr with nitroveratryl (Nvoc) chloride under basic conditions. 


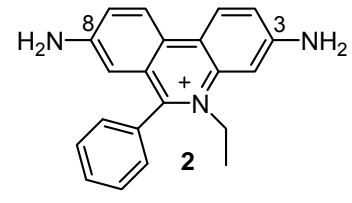

Nvoc-Cl DIEA, DMF<smiles></smiles>

Scheme 1. Synthesis of Nvoc-caged ethidium bromide @2.

As expected, (C2 did not show any fluorescence in the presence of a short double stranded DNA oligonucleotide, and competition experiments with uncaged $\mathrm{EtBr}$ confirmed that $\odot 2$ is unable to bind DNA. Irradiation of a solution of $\odot 2$ in Tris-HCl buffer; $100 \mathrm{mM} \mathrm{NaCl}$; $\mathrm{pH} 7.5$, with UV light (standard transilluminator lamp, $\lambda=300$ to $375 \mathrm{~nm}$ ), promoted the cleavage of the phololabile groups, thus regenerating the active EtBr intercalator (2). Importantly, this can be achieved in the presence of DNA, leading to a large increase (60-fold) in the emission intensity at $600 \mathrm{~nm}$, which is consistent with the intercalation of the released EtBr into the DNA (Figure 3).

HPLC monitoring of the UV uncaging showed that the process generates temporary Nvocmonoprotected intermediates. Control experiments with a mixture of specifically synthesized C3 and $\mathrm{C} 8$ Nvoc-monoprotected $\mathrm{EtBr}$ showed that these compounds are not fluorescent in presence of DNA, despite retaining some DNA binding affinity (see the supporting information, pages S9-S10). Therefore, uncaging of both groups is required for good DNA binding, and for DNA-dependent fluorescent staining. 


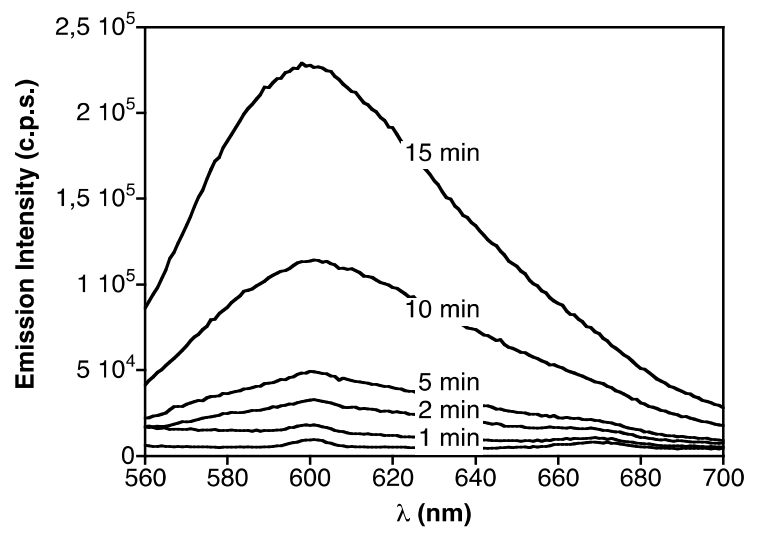

Figure 3. Fluorescence emission spectra of a $0.8 \mu \mathrm{M}$ solution of $\odot 2$ in Tris- $\mathrm{HCl}$ buffer at increasing irradiation times in the presence of a hairpin ds-oligonucleotide. No further increase in fluorescence is observed after $15 \mathrm{~min}$. irradiation; therefore shining light for 10-15 min is enough for complete uncaging.

Once the photocontrolled DNA binding of ethidium was confirmed, we studied whether the presence of the hydrophobic appendage could improve the cell transport properties of the dye. Therefore, independent samples of Vero cells were treated in parallel with $12.5 \mu \mathrm{M}$ caged $\odot 2$ and $20.0 \mu \mathrm{M}$ of $\mathrm{EtBr}(\mathbf{2})$, and examined by fluorescent microscopy after 30 min (excitation filter $530-550 \mathrm{~nm} / \mathrm{emission}$ filter $590 \mathrm{~nm}$ ). As shown in Figure $4 \mathrm{~A} / \mathrm{C}$, in both cases the cells were essentially non-fluorescent, and only a very faint emission could be detected in the case of EtBr. Remarkably, UV irradiation of the cells incubated with $@ 2 ~(10 \mathrm{~min})$ resulted in a very significant increase in the emission intensity in the cell nuclei (Figure 4B). ${ }^{19}$ This result must be correlated with photorelease of ethidium bromide from the inactive derivative @2. Therefore, in contrast to the parent phenantridinium 2, the designed photoresponsive derivative $@ 2$ seems to efficiently cross cell membranes, accumulating inside the cell in a latent form, until irradiation with UV light releases the active DNA binder. A control experiment at different irradiation times revealed a progressive increase in the fluorescence emission from the cells, reaching a maximum after approximately 10 min of irradiation (Figure 4, top). Interestingly, colocalization experiments with 
DAPI and mitotracker dyes show that the uncaged ethidium is mainly located in the nucleoli, therefore cannot be really considered as a true nuclear stain (See the supporting information, pages S15 and S16).
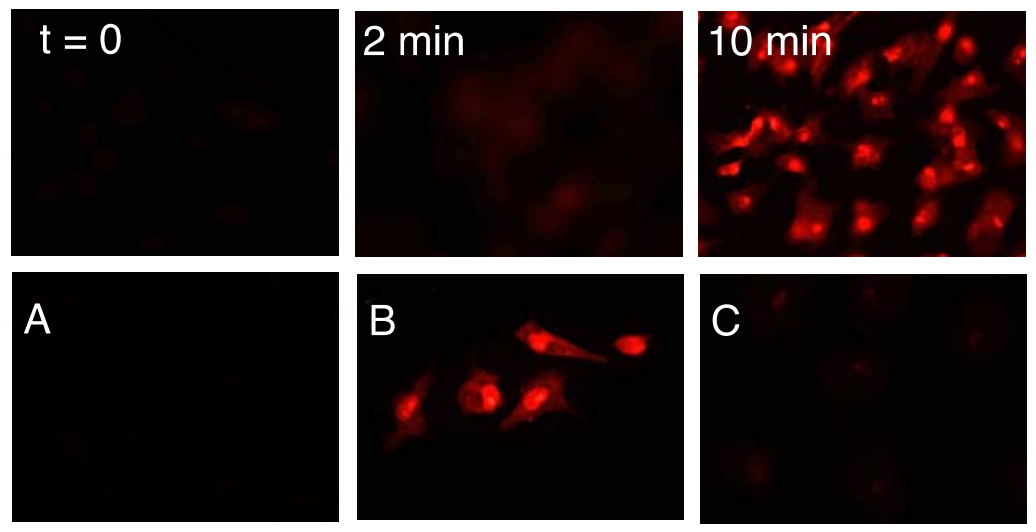

Figure 4. Top: Fluorescence microscopy of Vero cells (12.5 $\mu \mathrm{M} \odot 2)$ at increasing irradiation times. Bottom: $12.5 \mu \mathrm{M} \odot 2$, after 30 min incubation. A) before irradiation and uncaging; B) after uncaging by UV irradiation for $15 \mathrm{~min}$; C) control experiment with $20.0 \mu \mathrm{M}$ of ethidium bromide 2 after 30 min incubation. Pictures were taken with a Olympus DP-50 digital camera mounted on an Olympus BX51 microscope, equipped with U-MNG2 fluorescence mirror unit.

In addition to temporal activation, the caging strategy should also allow for the spatial control of the interaction. To demonstrate that, we prepared a cell monolayer on a $100 \mathrm{~mm}$ tissue culture plate (Figure $5 \mathrm{~A}$ ), and irradiated it through a cardboard cutout of our university acronym, USC. After 15 minutes of irradiation through the mask, we looked at the culture through a gel imaging system, observing the expected cellular photopatterning only for the cell populations that were irradiated (Figure 5B). Therefore, derivative $\odot 2$ is a light responsive, transport-efficient version of ethidium bromide, and should represent a significant addition to the yet short arsenal of nucleic acid stains that are effective in vivo. 

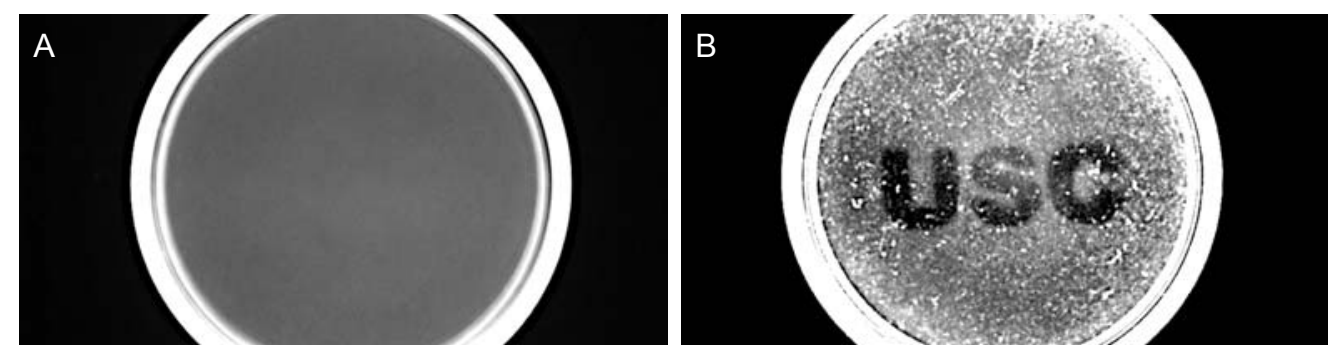

Figure 5. A) cell monolayer in the culture plate before irradiation; B) same culture after irradiation through a cardboard cutout.

The above results encouraged us to evaluate the performance of both caged DAPI (@1) and caged ethidium (@2) in a more stringent, therapeutically-relevant setting. Therefore we incubated mice eye retinas with $5.0 \mu \mathrm{M} \odot 1$ and $12.5 \mu \mathrm{M}$ of $\odot 2$ (PBS buffer) and analyzed the resulting cellular staining before and after irradiation. Before irradiation, no staining was visible, whereas after irradiation both $\subseteq 1$ and $\subseteq 2$ were effectively uncaged in the retina, and the cell nuclei became fluorescent (Figure $6 \mathrm{a}, 6 \mathrm{~b}$, see also time-lapse video $\mathrm{S} 2$ in the supporting information). Importantly, the same results were reproduced with human retinas (Figure 6d, 6e), confirming that these compounds can be activated in the complex cellular environment of human tissues.

In a step forward, we also explored the possibility of in vivo activation of these molecules by direct external irradiation of the eyes in living mice. Therefore, live mice were treated with caged DAPI (@1) using a femoral vein injection, and a transpupillar irradiation of one of their eyes was made. The mice were sacrificed and the retinas extracted and flat mounted for study. As expected, only the retina of the irradiated eyes showed a clear fluorescence emission, which was mostly concentrated in the vascular walls (Figure 6c), possibly because the blood-retinal barrier prevented most of the molecules to enter the retinal cells. ${ }^{20}$ These results confirm that the designed compounds can be selectively activated in the posterior segment of eyes of living 
animals using an external irradiation source, and suggest the eye as a suitable initial target organ for future therapeutic intervention using this type of light-activatable compounds. Delivery of drugs in a minimally invasive, safe and effective manner in the eye is still a major therapeutical challenge. ${ }^{21}$ Given the well-established use of lasers in ophthalmology, the uncaging tactic should open new opportunities to treat specific eye diseases that require the selective release of active drugs in specific areas of the eye. ${ }^{22,23}$

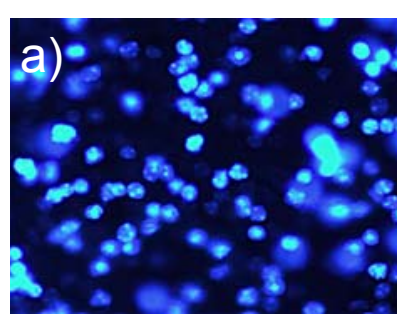

d)

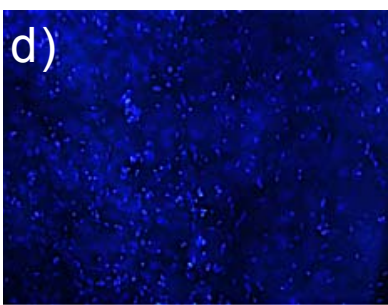

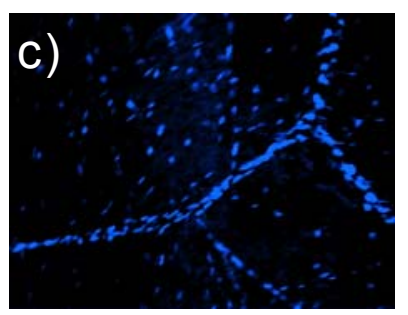
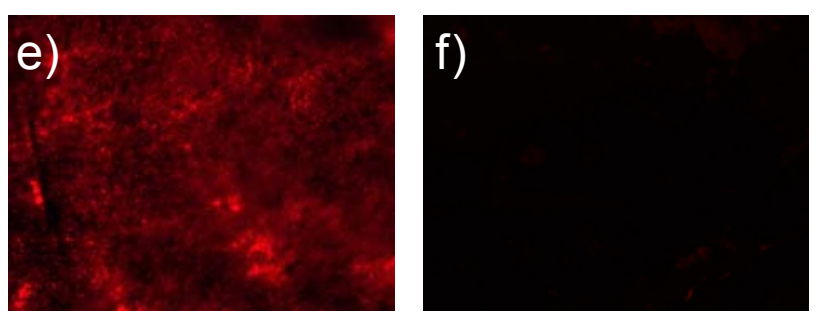

Figure 6.Fluorescence microscopy of mouse and human retinas. a) Mouse retina incubated with $5.0 \mu \mathrm{M}$ of@1, after $\sim 5$ min irradiation. b) Mouse retina incubated with $12.5 \mu \mathrm{M} \odot 2$, after $\sim 10 \mathrm{~min}$ irradiation. c) Retina extracted from a mouse previously injected with $@ 1$ in the right femoral vein $(100 \mu \mathrm{L}, 5.0 \mu \mathrm{M})$, and transpupillary irradiated for $\sim 10 \mathrm{~min}$ before sacrifying the animal; the nuclei of aligned cells forming a vessel image can be clearly observed. d) Human retina incubated with $5.0 \mu \mathrm{M} \odot \mathbf{1}$, after $\sim 5$ min irradiation. e) Human retina incubated with $12.5 \mu \mathrm{M}$ of (C2, after 10 min irradiation. f) Human retina incubated with $12.5 \mu \mathrm{M}$ of @2 before irradiation. Control, non-irradiation experiments were carried in allcases and none of them showed traces of fluorescence. Images $a$ and $b$ were acquired with $1 \mathrm{~s}$ exposure time, images $c$-f with $400 \mathrm{~ms}$. All images with ISO 400 sensitivity.

In conclusion, we have demonstrated that the Nvoc caging strategy can be applied to classic 
DNA intercalators like EtBr. Since these molecules are nucleic acid-dependent fluorescent probes, they provide an excellent opportunity for real-time monitoring of the activation process in living cells. For the Nvoc-DAPI (@1), we could observe the uncaging process and cell redistribution of the released stain in real time. Moreover, the caging strategy can be exploited not only for the "on demand" spatiotemporal control of the DNA-binding, but also for fine-tuning the physicochemical properties of the substrates. In the case of the Nvoc-caged derivative $\subseteq 2$, this allows for a rapid cell internalization, overcoming the poor cell transport ability of the parent ethidium bromide. Importantly, we have also demonstrated that this strategy can be successfully implemented in therapeutic-relevant tissues such as the retina, being even possible to activate them with external irradiation in the eyes in living mice. To the best of our knowledge, our results provide the first evidence on the use of caged compounds in eyes, and demonstrate that focalized release of drugs in the eye can be made with a minimally invasive technique, opening a new door for therapeutic and perhaps diagnostic applications.

\section{Materials/methods}

General: All reagents were acquired from commercial sources. Reactions were followed by analytical RP-HPLC with an Agilent 1100 series LC/MS using an Eclipse XDB-C18(4.6 x 150 $\mathrm{mm}, 5 \mu \mathrm{m}$ ) analytical column. Standard conditions for analytical RP-HPLC consisted on a linear gradient from $5 \%$ to $95 \%$ of solvent $B$ for $30 \mathrm{~min}$ at a flow rate of $1 \mathrm{~mL} / \mathrm{min}$ (solvent $\mathrm{A}$ : water with $0.1 \%$ TFA, solvent B: acetonitrile with $0.1 \%$ TFA). Compounds were detected by UV absorption at 220, 270, 304 and $330 \mathrm{~nm}$. Final products were purified on a Büchi Sepacore preparative system consisting on a pump manager $C-615$ with two pump modules $C-605$ for binary solvent gradients, a fraction collector C-660, and UV Photometer C-635. Purification was made using reverse phase of water/acetonitrile $0.1 \%$ TFA, using a pre-packed preparative cartridge $(150 \mathrm{x}$ 
$40 \mathrm{~mm}$ ) with reverse phase $\mathrm{RP}_{18}$ silica gel (Büchi order \#54863). The fractions containing the products were freeze-dried, and their identity was confirmed by ESI-MS(+). NMR spectra were recorded using Varian Mercury 300orBrucker DPX 250 spectrophotometers, and processed using the MestReNova v6.1.1-6384 suite (Mestrelab Research). ${ }^{1} \mathrm{H}$ NMR spectra were processed applying Global Spectrum Deconvolution (GSD).

\section{Synthetic procedures}

\section{3,8-bis(\{[(4,5-dimethoxy-2-nitrobenzyl)oxy]carbonyl\}amino)-5-methyl-6-}

phenylphenanthridinium ( $\mathrm{NvOC}_{2}$-ethidium, $\mathrm{C}_{2}$ ). To a solution of commercially available ethidium bromide (2), (100 mg, $0.253 \mathrm{mmol})$ in $25 \mathrm{~mL}$ of DIEA/DMF (0.195 M) was added Nitroveratryl chloride $(209 \mathrm{mg}, 0.759 \mathrm{mmol})$. The resulting mixture was stirred for $16 \mathrm{~h}$ under $\mathrm{Ar}$ and in the dark. After concentration, the residue was purified by preparative reverse-phase chromatography (Büchi Sepacore) (5 min isocratic 15\% B, followed by linear gradient from 15\% to $95 \%$ B 30 during $\mathrm{min}$, and $10 \mathrm{~min}$ isocratic at $95 \% \mathrm{~B}$ ). The combined fractions were concentrated and freeze-dried to obtain the desired product (๑2) as a trifluoroacetic salt $(77 \mathrm{mg}$, $0.086 \mathrm{mmol}, 34 \%)$.

In vitro fluorescence experiments with $\odot 2:$ A fluorescence cuvette containing a $0.82 \mu \mathrm{M}$ solution of $@ 2$ in Tris- $\mathrm{HCl}$ buffer $20 \mathrm{mM} ; 100 \mathrm{mMNaCl}$; $\mathrm{pH} 7.5$, and $0.136 \mu \mathrm{M}$ hairpin DNA oligonucleotide AACGTT was irradiated with UV light, and fluorescence emission spectra were recorded after different irradiation times. Hairpin oligonucleotide: AACGTT: GGC AAGCTT CGC TTTTT GCG AAGCTT GCC.

Cell uptake experiments. Vero cells were maintained in DMEM (Dulbecco Modified Eagle Medium) containing $10 \%$ of FBS (Fetal Bovine Serum). The day before the cellular uptake experiments, cells were seeded in twelve well plates containing glass coverslips $(15 \mathrm{~mm})$. Cells were then washed 3 times in PBS and overlaid with $1 \mathrm{~mL}$ of fresh PBS with no serum added. 
Compound $\odot 1$ was added in order to obtain a final concentration of $5.0 \mu \mathrm{M}$, and in the case of (C2 $12.5 \mu \mathrm{M}$. Samples were incubated for $30 \mathrm{~min}$ at room temperature in the absence of light. For video recording and snapshots of Figure 2, after incubation the coverslips were mounted on glass slides with Mowiol4-88® [100 mg/mL in $100 \mathrm{mM}$ Tris- $\mathrm{HCl} \mathrm{pH} 8.5,25 \%$ glycerol and $0.1 \%$ DABCO (as an antifading agent)]. Irradiation of selected samples was performed with a standard gel UV transilluminator (8 watt, $\lambda$ exc $300-370 \mathrm{~nm}$ ). Images were obtained with an Olympus DP50 (Olympus Corp., Tokyo, Japan) digital camera mounted on an Olympus BX51 fluorescence microscope (Olympus BX51, Olympus Corp., Tokyo, Japan) equipped with a 360-370 nm excitation filter, $420 \mathrm{~nm}$ emission filter for the cells treated with $₫ 1$ and were further processed (cropping, resizing and contrast global contrast and brightness adjustment) with Adobe Photoshop (Adobe Systems). In the case of @2 we used a 530-550 nm excitation filter, and a $590 \mathrm{~nm}$ emission filter.

Experiments with retinas and with animals. Animal retinas were obtained from 3 month-old wild type SV129 mice originally adquired from Charles River Laboratories (Boston, Massachussetts, USA). The animals were terminally anesthetized, the eyes enucleated, the retinas dissected from the eyecup, and flat mounts prepared with the ganglion cell layer uppermost. Then they were incubated with a solution of caged ethidium (@) 2, $12.5 \mu \mathrm{M}$ in PBS buffer), or caged DAPI (@1, $5.0 \mu \mathrm{M}$, PBS buffer). The uncaging was performed with a standard gel UV transilluminator for $\odot 2(\lambda=300$ to $375 \mathrm{~nm})$. In the case of $\odot 1$, it was found that the light of the microscope (Olympus BX51) was intense enough to cleave the photolabile groups. The irradiated samples were observed in a fluorescence microscope (Olympus BX51, Olympus Corp., Tokyo, Japan) equipped with a 530-550 nm excitation filter, $590 \mathrm{~nm}$ emission filter for the cells treated with $\odot 2$, and a 360-370 nm excitation filter, $420 \mathrm{~nm}$ emission filter for the cells treated with $@ 1$. Pictures were taken with an Olympus DP-50 camera (Olympus Corp., Tokyo, Japan) mounted on the microscope. The same procedure was followed in the case a human 
retina obtained from a patient who underwent ocular evisceration because of a painful terminal glaucoma. Once the retina was removed from the eye, it was placed in DMEM (Dulbecco Modified Eagle Medium) containing $10 \%$ of FBS (Fetal Bovine Serum), 1\% penicillin/streptomycin, and 1\% glutamate, and cut into several pieces. Each piece was placed on a microscope slide and the incubation procedure described above was followed. Consent permission was obtained from the patient and the procedure was authorized by the Ethical Committee for Clinical Research of our local government (Xunta de Galicia).

For in vivo experiments the animals were anesthesized by intraperitoneal injection of $4 \%$ chloral hydrate (400 mg/kg body weight) and a drop of tropicamide solution $(10 \mathrm{mg} / \mathrm{ml})$ was instilled on the eyes to produce mydriasis. The right femoral vein was canulated, and $0.1 \mathrm{ml}$ of a solution of caged DAPI (@1, $5.0 \mu \mathrm{M}$ in PBS) was injected. Then, the right eye was transpupillary irradiated $(\lambda=300$ to $375 \mathrm{~nm})$. Finally, the animals were sacrificed by cervical dislocation, the eyes were enucleated, and the retinas mechanically dissected out were mounted and viewed in the fluorescent microscope. All experiments involving animals were conducted according to the Bioethical Committee of our institution and adhered to the ARVO statement for the Use of Animals in Ophthalmic and Vision Research.

\section{Acknowledgments}

We thank the support given by the Spanish grants SAF2007-61015, SAF2010-20822-C02, CTQ2009-14431/BQU, ConsoliderIngenio 2010 CSD2007-00006, and the Xunta de Galicia INCITE09 209 084PR, GRC2010/12, PGIDIT08CSA-047209PR. M.I.S. thanks the Spanish Ministry of Education for FPU PhD fellowships. 
References

[1] Praveen, B. S., Reddy, S., Sonhdi, S. M., and Lown, J. W. (1999) Synthetic DNA minor groove-binding drugs. Pharmacol.Ther. 84, 1-111.

[2] Boer, D. R., Canals, A, and Coll, M. (2009) DNA-binding drugs caught in action: the latest 3D pictures of drug-DNA complexes.J. Chem. Soc., Dalton Trans. 3, 399-414.

[3] Strekowski, L., and Wilson, B. (2007) Noncovalent interactions with DNA: an overview. Mutat. Res. 623, 3-13

[4] Pazos, E., Mosquera, J.; Vázquez, M. E., and Mascarenas, J. L. (2011) DNA Recognition by Synthetic Constructs.ChemBiochem.12, 1958 - 1973.

[5] Vázquez, M. E., Caamaño, A., and Mascareñas, J. L. (2003) From transcription factors to designed sequence-specific DNA-binding peptides. Chem. Soc. Rev. 32, 338-349.

[6] Deiters, A. (2010) Principles and Applications of the Photochemical Control of Cellular Processes. ChemBioChem 11, 47-53.

[7] Lee, H.-M., Larson, D. R., and Lawrence, D. S. (2009) Illuminating the Chemistry of Life: Design, Synthesis, and Applications of "Caged" and Related Photoresponsive Compounds. ACS Chem. Biol. 4, 409-427.

[8] Caamaño, A. M., Vázquez, M. E., Martínez-Costas, J., Castedo, L., and Mascarenas, J. L. (2000) A Light-Modulated Sequence-Specific DNA-Binding Peptide. Angew.Chem. Int. Ed. 39, $3104-3107$.

[9] Prasad, P.N. Introduction to Biophotonics.Wiley-Interscience; 1 edition, 2003.

[10] Wilson, W. D., Tanious, F. A., Barton, H. J., Jones, R. L., Fox, K., Wydra, R. L., and Strekowski, L. (1990) DNA sequence dependent binding modes of 4',6-Diamidino-2phenylindole (DAPI). Biochemistry 29, 8452-8461.

[11] Waring, M. J. (1965) Complex formation between ethidium bromide and nucleic acids.J. Mol. Biol.13, 269-282.

[12] LePecq, J. B., and Paoletti, C. (1967) A fluorescent complex between ethidium bromide and nucleic acids. J. Mol. Biol. 27:87-106.

[13] Vázquez, O., Sánchez, M. I., Martínez-Costas, J., Vázquez, M. E. and Mascareñas, J. L. (2010) Bis-4-aminobenzamidines: Versatile, Fluorogenic A/T-Selective dsDNABinders. Org. Lett. 12, 216-219.

[14] Vázquez, O., Sánchez, M. I., Mascareñas, J. L. and Vázquez, M. E. (2010) dsDNAtriggered energy transfer and lanthanide sensitization processes. Chem. Commun. 46, 5518-5520. 
[15] Sánchez, M. I., Vázquez, O., Vazquez, M. E., and Mascareñas, J. L. (2011) Lightcontrolled DNA binding of bisbenzamidines.Chem. Commun. 47, 11107-11109.

[16] Yasumura, Y., and Kawakita, Y. (1963) Studies on SV40 in tissue culture - Preliminary step for cancer research "in vitro." Nihon Rinsho 21,1201-1215.

[17] Bhal, S.K., Kassam, K., Peirson, I.G., and Pearl, G.M. (2007) The rule of five revisited: applying LogD in place of LogP in drug-likeness filters. Mol. Pharmaceutics 4, 556-560.

[18] Lusk, C. P., Blobel, G., and King, M. C. (2007) Nuclear pores allow small molecules (<5 $\mathrm{kDa}$ ) to freely diffuse across the nuclear envelop. Highway to the inner nuclear membrane: rules for the road. Nature Rev. Mol. Cell Biol. 8, 414-420.

[19] Control experiments demonstrate that these short irradiation times with the transilluminator lamp do not compromise the cell viability, see the supporting information, page S13.

[20] This is a known phenomenon that occurs in healthy retinas, but if the retinas present some degree of damage the compounds should be able to enter the cells: Cunha-Vaz, J. G. (1997) The blood-ocular barriers: past, present, and future. Doc Ophthalmol 93, 149-157.

[21] Janoira, K. G., Gunda, S., Boddu, S. H., and Mitra, A. K. (2007) Novel approaches to retinal drug delivery. Expert Opin Drug Deliv 4, 371-388.

[22] Thrimawithana, T. R, Young, S., Bunt, C. R., Green, C., and Alany, R. G. (2011) Drug delivery to the posterior segment of the eye. Drug Discovery Today 16, 270-277.

[23] Gomez-Ulla.F., Gonzalez, F., and Torreiro, M. G. (1998) Diode laser photocoagulation in idiopaticpolypoidalvasculopathy. Retina 18, 481-483. 